Publisher homepage: www.universepg.com, ISSN: 2663-7804 (Online) \& 2663-7790 (Print)

https://doi.org/10.34104/ajeit.021.073081

Australian Journal of Engineering and Innovative Technology

Journal homepage: www.universepg.com/journal/ajeit

\title{
Detection and Implementation of Blood Group and Hb Level by Image Processing Techniques
}

\author{
Kh. Khaleduzzaman ${ }^{1}$, Md. Hasan Mahmud ${ }^{1}$, Tarun Debnath ${ }^{1}$, Sohag Sarker ${ }^{1 *}$ and Pallab Kanti Podder ${ }^{1}$ \\ ${ }^{1}$ Department of Information and Communication Engineering, Pabna University of Science and Technology, Pabna-6600, \\ Bangladesh. \\ *Correspondence: sohagsarker5614@gmail.com (Sohag Sarker, Assistant Professor, Department of Information and Com- \\ munication Engineering, Pabna University of Science and Technology, Pabna-6600, Bangladesh).
}

\begin{abstract}
In an emergency, an urgent blood transfusion from a person to the patient is required and blood group identification is the first process to do so. In addition, a hemoglobin test is often required to make decisions about blood transfusion as well as to check anemia. Hemoglobin testing is also required for complete blood count and monitoring a number of diseases. These blood tests are almost difficult in rural areas where lab facilities are not sufficient. Researchers proposed a number of methods to identify blood groups using computer vision techniques. However, no study was conducted to identify blood group and hemoglobin level in a work using image processing techniques and an android mobile application which shows high detection accuracy. In this paper, manual clinical experiments have been replaced by an android app using image processing techniques to detect blood groups and hemoglobin levels except users require using antigen before taking samples. The proposed technique is divided into two portions. The first portion is blood group detection, which is done by taking a blood sample and performing the grayscale conversion, binary conversion, segmentation, edge detection, and computation to make the decision. The second section describes how to determine hemoglobin levels by comparing a blood sample image to a hemoglobin color scale (HCS). Here, the Hemoglobin value is determined from their RGB values. It has been discovered that the proposed approaches are capable of detecting hemoglobin levels and blood groups in a cost-effective and error-free manner. As a result, the tests can be conducted in a remote area without adequate lab facilities and the proposed work can solve major steps in blood transfusion difficulties and anemia.
\end{abstract}

Keywords: HCS, Agglutination, Anemia, Image segmentation, Edge detection, ABOblood type and Grouping.

\section{INTRODUCTION}

Blood is a composition of red blood cells, white blood cells, and platelets in liquid plasma. Blood groups are identified by antibodies and antigens present in plasma. There are four blood groups i.e. A, B, AB, and $\mathrm{O}$ (NHS, 2020). If the blood is agglutinated by the presence of Rh-D antigen, the group of the blood is posi-

UniversePG I www.universepg.com tive, otherwise negative (K et al., 2016; Dong et al., 2017). This blood groups identification is often required in case of blood transfusion. The identification of the blood group is not enough at all. A hemoglobin test helps to decide blood transfusion. In addition, a lack of hemoglobin leads to Anemia. Anemia is a type of disease in which a person lacks enough red blood 
cells for carrying necessary oxygen for soft body tissues. Pregnant women, as well as young children, are the most vulnerable to its devastating effects (Kharkar et al., 2013). So, the blood group detection and hemoglobin test are often required in case of any emergency and these tests are conducted manually in a laboratory. Unfortunately, the problem arises for the people who live in rural areas. The scarcity of enough lab facilities and trained personnel are often noticed. To address these problems, researchers have proposed a lot of techniques to identify blood group and hemoglobin $\mathrm{Hb}$ levels to support those people in case of emergency. The authors in (Ravindran et al., 2017) have proposed image processing techniques to deter-mine and classify blood types. In the experiment, slide testing is done using thresholding and morphological gradient operations. This developed method shows greater accuracy. Authors in (Dong et al., 2017) proposed another image processing technique based on feature extraction by exploiting Niblack segmentation, Otsu algorithm to identify the ABO blood group. The most extensively used blood grouping system is $\mathrm{ABO}$, which was invented by Austrian Rand Steiner. It provides accurate results but consumes a lot of time, which is the main constraint of this technique. Authors in (Dhande et al., 2018) proposed a cost-effective technique using an image processing technique for blood typing. The key technologies in the research are morphological operations, erosion, dilation, and thres holding. The only limitation is observed in the research is that the research does not identify the $\mathrm{Rh}$ factor of the blood. In another research, blood grouping and typing are done using image processing, conveyor belt, and digital microscope (Azam et al., 2020; Jayakumar et al., 2020).

This technique can process a lot of samples at a time in the laboratory but it does not proceed to a process of testing blood outside of laboratories such as in rural areas or on mountain trails. Our proposed methodology would be capable to complete blood grouping or typing without any help from a medical technician so that major steps in blood transfusion problems can be eradicated. In addition, Anemia is a major problem commonly found in women. A study shows that $77 \%$ of the female workers among the total garments workers suffer from different grades of anemia in
Bangladesh (Khatun et al., 2013). In this circumstance, sophisticated low-cost $\mathrm{Hb}$ testing equipment must be developed. Authors in (Ahsan et al., 2017) proposed $\mathrm{Hb}$ level detection technique from video images. The limitation is that a mobile application is used to collect the required video but the detection process needs computer analysis. Mobile application only enhances the video. Authors in (Zafar et al., 2018) investigated ultra-compact plasmonics-based sensors with fano resonance. The prototype's applicability to detect $\mathrm{Hb}$ concentration was investigated using metal-insulator-metal (MIM) waveguide geometry. Authors in (An et al., 2019) developed a microchip electrophoresis named HemeChip+ to identify anemia and hemoglobin variants. One observation is found that the HemeChip+ single-use cartridge is a good solution but it requires Gazelle (Display analyzer by Hemex Health Inc.). From these studies, some observations are found these are: most of the bloods grouping techniques are not applicable in rural and remote areas, some tests require additional equipment which can be afforded in a lab environment but in case of rapid test this equipment cannot be afforded. No study was conducted for combing the blood grouping and $\mathrm{Hb}$ test in a combined cost-effective manner. By addressing these limitations, this paper proposes a high efficient blood grouping and $\mathrm{Hb}$ level testing by exploiting image processing mechanisms through an android smart phone.

The testing procedure does not require an additional instrument. It only requires $\mathrm{A}, \mathrm{B}$, and $\mathrm{D}$ antigens for blood testing and a test strip for $\mathrm{Hb}$ level testing. Blood grouping and $\mathrm{Hb}$ level testing are carried out in this work using an image processing method included in an Android mobile application. The initial step is to take a blood sample, after which the slide testing procedure is considered. In this scenario, three drops of blood are mixed with antigens $\mathrm{A}, \mathrm{B}$, and $\mathrm{D}$ by placing them serially. The captured image is then processed through a variety of stages, including grayscale conversion, binary conversion, image segmentation, edge detection, and edge calculation. After these series of processing, a blood group is detected. In the case of $\mathrm{Hb}$ level detection we consider, World Health Organization (WHO)'s HCS method. The WHO HCS method is a low-cost color-scale method 
for measuring hemoglobin concentration from a drop of blood (Kumar et al., 2014; Lewis et al., 1998; Stott et al., 1995). The hemoglobin detection process of this work is inspired by the manual HCS method and it is taken over by an automatic android app-based detection system composed of several image processing techniques. Such as RGB value comparison, color intensity calculation, background removal, and result in analysis. The proposed mechanism shows an optimal accuracy for blood grouping and $\mathrm{Hb}$ level detection. It has a hundred percent accuracy in blood grouping and maximum accuracy in $\mathrm{Hb}$ level measurement.

\section{ABO Group Classification}

The schematic representation of the blood group detection technique is shown in Fig 1. The blood sample that will be analyzed has to take over a slide. The grouping is decided from when antibodies of blood cells make sure the reaction with erythrocyte antigens i.e. agglutination. The antigen response over antibody is shown in Table 1.

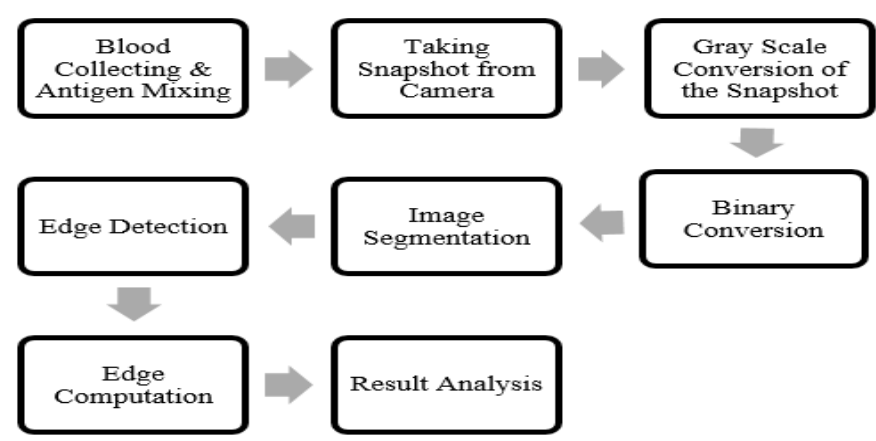

Fig 1: Block diagram of working procedure for detecting Blood Group.

Table 1: Antigen response over antibody of a blood cell.

\begin{tabular}{|c|c|c|c|}
\hline Anti -A & Anti -B & Anti -D & Type \\
\hline N & N & N & O - \\
\hline N & N & Y & O + \\
\hline N & Y & N & B - \\
\hline N & Y & Y & $\mathrm{B}+$ \\
\hline Y & N & N & $\mathrm{A}-$ \\
\hline Y & N & Y & $\mathrm{A}+$ \\
\hline Y & Y & N & $\mathrm{AB} \mathrm{-}$ \\
\hline Y & Y & Y & $\mathrm{AB} \mathrm{+}$ \\
\hline
\end{tabular}

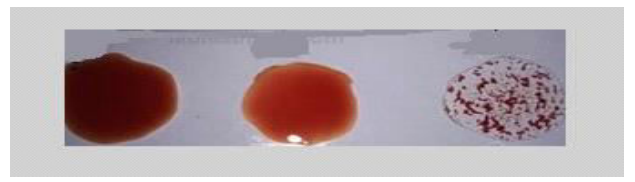

(a)

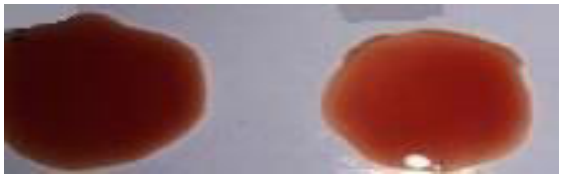

(b)

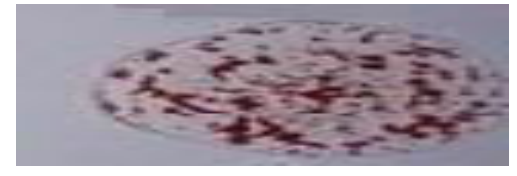

(c)

Fig 2: Blood Sample after mixing antigens, (a) Original image; (b) No Agglutination; and (c) Agglutination.

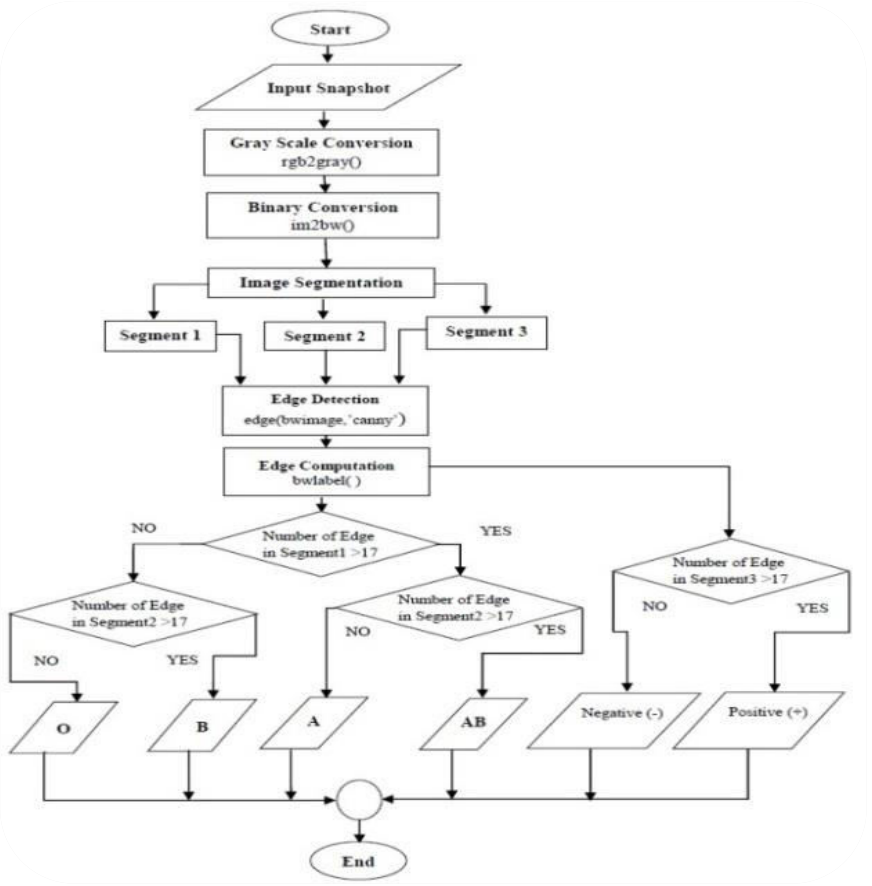

Fig 3: Flow chart for detecting ABO group type.
' $\mathrm{N}$ ' means there is no reaction occurs between antigen and antibody i.e. no agglutination and ' $\mathrm{Y}$ ' represents the agglutination that occurs. Image is taken from the blood sample slide by image acquisition device and the image after being mixed with antigen is shown in Fig 2(a). The occurrence of non-agglutination and agglutination of blood is respectively shown in Fig 2(b) and 2(c). To figure out and analyze the reaction between antigen and reagent by image processing the several methods like segmentation, edge detection, the calculation is used, and then the grouping decision is made from Table $\mathbf{1 .}$ The input image is followed by the flow chart procedures for ABO-type blood grouping shown in Fig 3. Firstly, the grayscale conversion of the input image is done through binary conversion then segmentation. Hence edge is detected and derived from these segments.

The flow chart finally ends with the decision-making 
process. Grayscale conversion is one of the fundamental approaches for converting RGB color images into a grayscale image. The basic method of this transformation is taking the contribution of red, green, and blue intensity partially. In comparison to the average method, the Luminosity method produces better grayscale conversion results. In equation (1), $r, g, b$ denotes the intensity value of red, green, blue respectively of the color image and $g(x, y)$ is the grayscale image. In

Fig 4, the gray scale image of Fig 2(a) is shown.

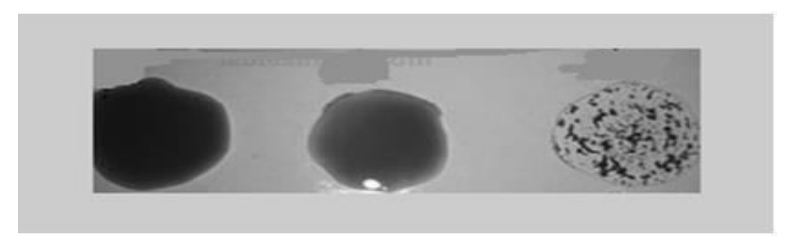

Fig 4: Gray scale image of Fig 1(a).

A threshold is employed in the transformation of a grayscale image to binary. The variety of intensity 0-255 of gray image is mapped into 0-1 i.e. binary image shown in Fig 5. This is done by accepting a custom threshold value $T$ and producing a binary image as an output, $b(x, y)$.

$$
\begin{gathered}
g(x, y)=0.299 * r+0.587 * g+0.114 * b \\
b(x, y)=\left\{\begin{array}{l}
1, \text { if } g(x, y) \geq T \\
0, \text { otherwise }
\end{array}\right.
\end{gathered}
$$

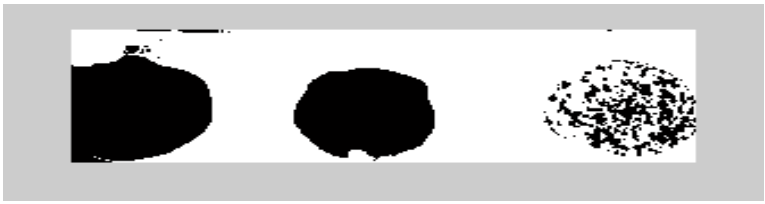

Fig 5: Binary image from gray scale image.

A method of splitting an image into different parts is called segmentation (Vala et al., 2013). The converted binary image, $b(x, y)$ is divided vertically into three block segments i.e. $s_{1}(x, y), s_{2}(x, y)$, and $s_{3}(x, y)$. The original image's number of columns determines how many segments are needed. In equation (4), (5) and (6), $i$ denotes the number of columns of each block, Fig 6 shows the three segments of the binary image. Canny image detection is a technique of detecting an edge from the binary image, having a Gaussian filter to reduce noise and provides the smooth image as output Fig 7 shows the canny edge detection from a binary image.

UniversePG I www.universepg.com

$$
i=\frac{\text { no. of coumn of } b(x, y)}{3}
$$$$
\left[\begin{array}{cccc}
b(0,0) & b(0,1) & \cdots & b(0, N-1) \\
b(1,0) & b(1,1) & \cdots & b(1, N-1) \\
\cdots & \cdots & \cdots & \cdots \\
b(M-1,0) & b(M-1,1) & \cdots & b(M-1, N-1)
\end{array}\right]
$$

$$
\left[\begin{array}{cccc}
b(0,0) & b(0,1) & \cdots & b(0, i-1) \\
b(1,0) & b(1,1) & \cdots & b(1, i-1) \\
\cdots & \cdots & \cdots & \cdots \\
b(M-1,0) & b(M-1,1) & \cdots & b(M-1, i-1)
\end{array}\right]
$$

$$
\left[\begin{array}{cccc}
b(0, i) & b(0, i+1) & \cdots & b(0,2 i-1) \\
b(1, i) & b(1, i+1) & \cdots & b(1,2 i-1) \\
\cdots & \cdots & \cdots & \cdots \\
b(M-1, i) & b(M-1, i+1) & \cdots & b(M-1,2 i-1)
\end{array}\right]
$$

$$
\left[\begin{array}{cccc}
b(0,2 i) & b(0,2 i+1) & \cdots & b(0, N-1) \\
b(1,2 i) & b(1,2 i+1) & \cdots & b(1, N-1) \\
\cdots & \cdots & \cdots & \cdots \\
b(M-1,2 i) & b(M-1,2 i+1) & \cdots & b(M-1, N-1)
\end{array}\right]
$$

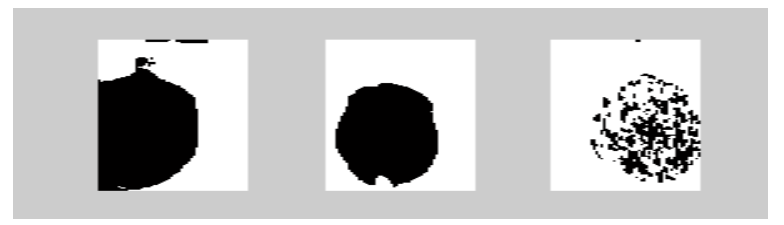

Fig 6: Segmentation of binary image.

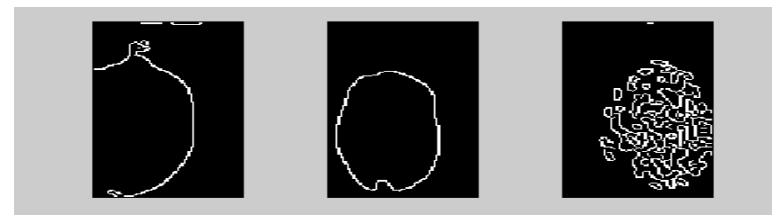

Fig 7: Canny edge detection from binary image.

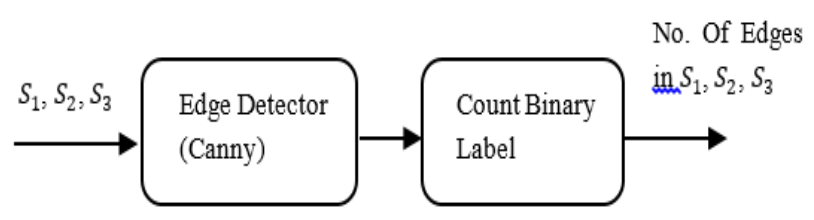

Fig 8: Edge computation process.

After detection process is done the edges are counted from the total number of connected objects found in that binary image. The edge computation process is illustrated in Fig 8.

\section{Hemoglobin (Hb) Detection Process}

HCS is a simple, accurate and cheap quantitative method to assess hemoglobin concentration outside of 
the laboratory, containing six different hues of color, which refers $4,6,8,10,12,14 \mathrm{~g} / \mathrm{dL}$ values of hemoglobin related to the blood sample (Marn et al., 2016; Hasan et al., 2021). Fig 9 shows the WHO HCS. The hemoglobin estimation is illustrated in the Fig 10. A drop of blood is placed on the test strip, and after about 30 seconds, the blood sample's color matches one of the hues on the HCS. The Hemoglobin level of a blood sample is estimated based on the closest match with HCS. Our goal is to take a photograph of

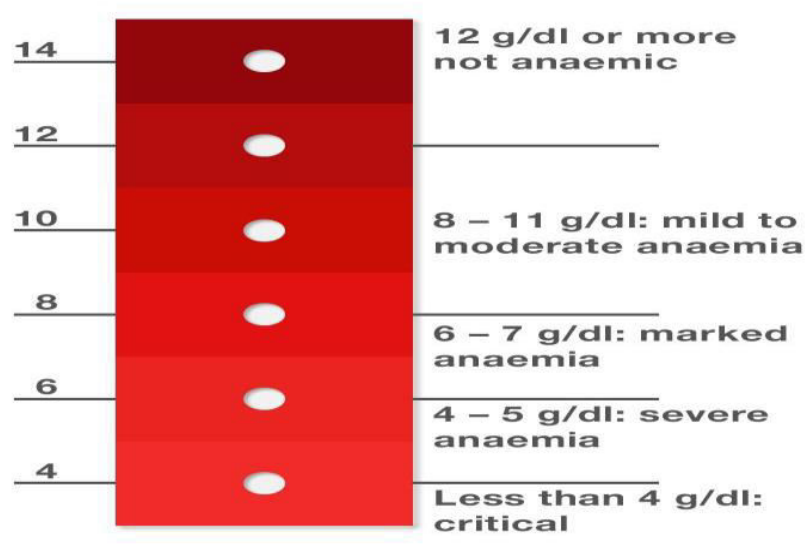

Fig 9: WHO hemoglobin color scale (HCS).

\section{Android Application Layout}

The recommended methodology is translated into an android application so that people in remote regions can benefit from it. Moreover, the application will provide some real blood groups and $\mathrm{Hb}$ test reports. Using Android Studio, an .apk file is created initially. Then the android application is installed in an android. A both HCS and blood samples, and it's also important to make sure that both images are in the same environment i.e. same distance, angle, illumination, resolution, etc. thus its monotonicity remains constant. At least 5MP Smart Phone Camera, 90-degree camera angle, a distance of around $7.5 \mathrm{~cm}$, and illumination above 500 lux in natural outdoor light has been regarded as a standard for our work which provides a comparatively high inter-cluster distance (ICD) and the monotonicity is maintained too.

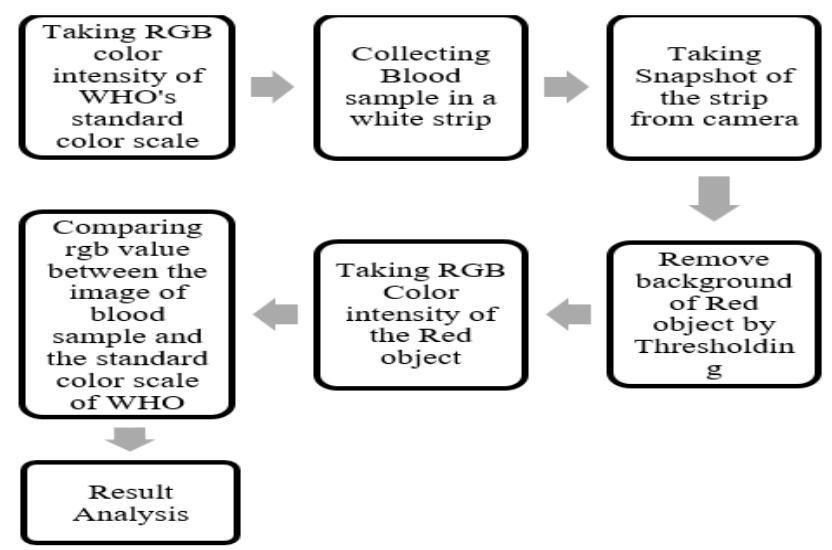

Fig 10: Working procedure of determining Hemoglobin level.

user interface, some layouts will be opened at the moment of usage. These layouts are described here: After opening the mobile app or android application the user's "Home Layout" will be displayed (Fig 11). Two buttons are available in the home layout. If the user wants to check the $\mathrm{Hb}$ level, he/she just needs to touch the 'Test Hemoglobin level' button.

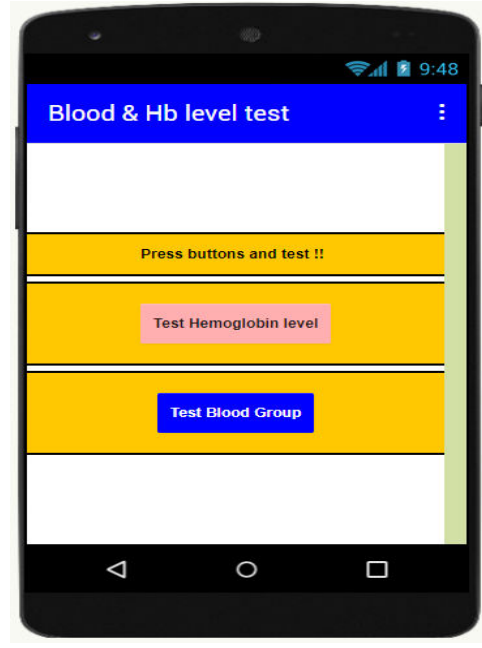

Fig 11: Home Layout.
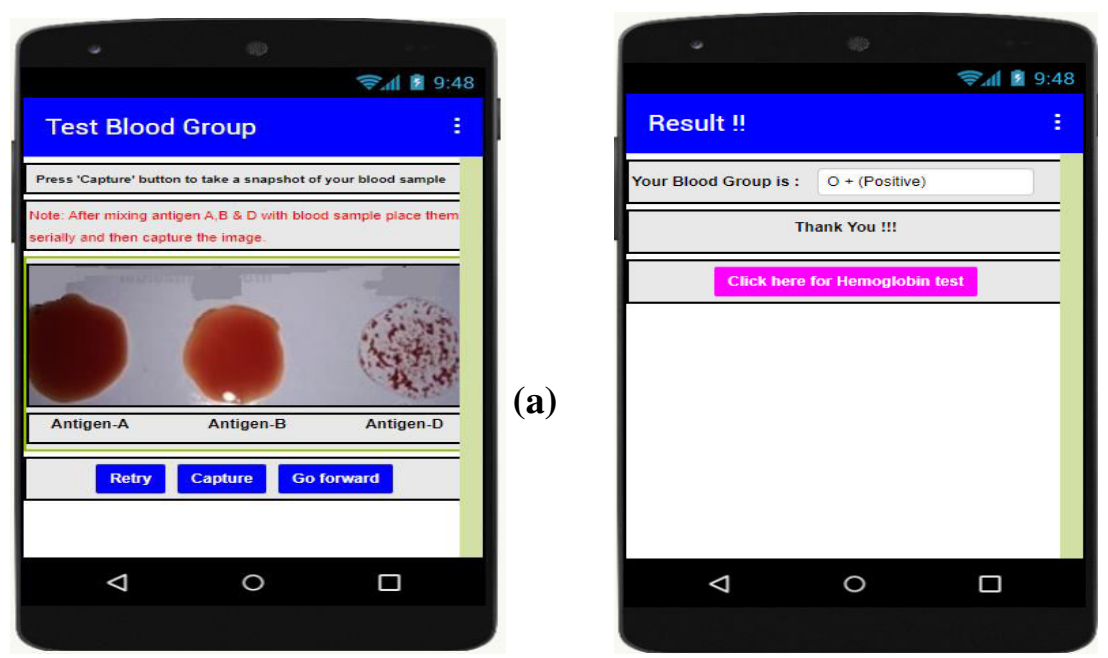

(a)

(b)

Fig 12: (a) Image Capture layout for Blood group detection, (b) Blood group testing result. 

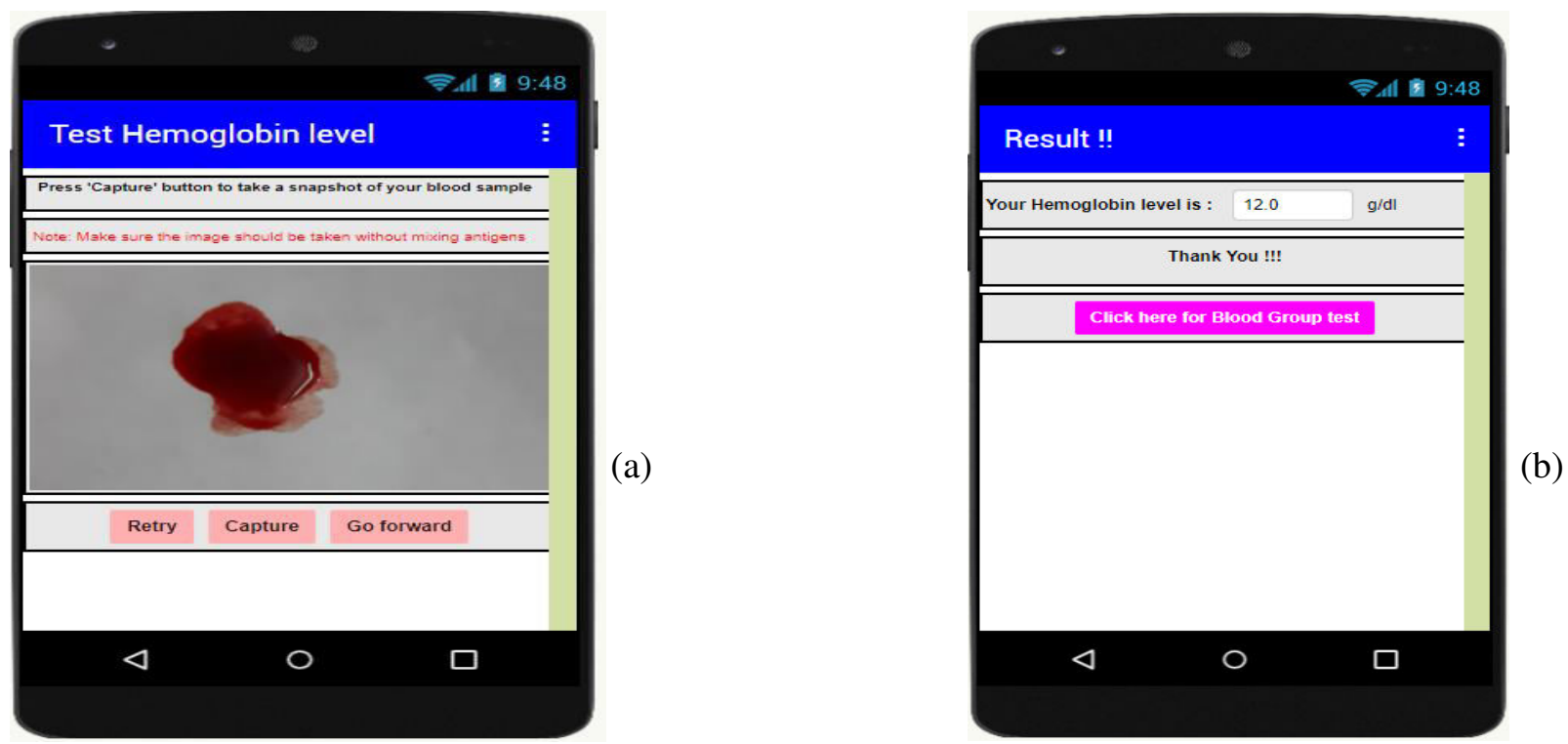

Fig 13: (a) Image capture layout for $\mathrm{Hb}$ level testing; (b) Hb testing result.

If the user needs blood group information, he/she just needs to touch the 'Test Blood Group' button. Consider the case of a user who wants to know his/her blood group. Then he/she will be required the A, B, D antigens and blood samples of interest. It must be ensured that mixing the antigens with the blood samples must be aligned sequentially (Antigen-A, Antigen-B, and Antigen-D) on a glass slide as depicted in Fig 12(a). Then the report layout will be generated after touching the 'Go forward' button as an immediate effect which is depicted in Fig 12(b). Let the user check the $\mathrm{Hb}$ level, he/she just needs to touch the 'Test Hemoglobin level' button from Home Layout.

For testing $\mathrm{Hb}$, the user just needs a test strip. After collecting the blood sample, it needs to be placed on the test strip and allowed to dry for 30 seconds. Then we just need to capture the image from the Test Hemoglobin Level for taking the image shown in Fig 13(a). If the proper image is captured then the 'Go forward' button will be visible for the next action. Finally, the user will have the ability to see his level of hemoglobin in the layout after touching the 'Go forward' button which is shown in Fig 13(b).

\section{RESULTS AND DISCUSSION:}

Based on our research of about 50 samples, a number of edges greater than 17 indicate agglutination, whereas a number less than 17 indicates the absence of agglutination. Table 2 displays the experimental data of UniversePG I www.universepg.com five different samples. $N_{A}, N_{B}$, and $N_{D}$ represent edge count for segments $S_{1}, S_{2}$, and $S_{3}$ respectively (Fig 8 ).

Table 2 shows that the edge value of $\mathrm{N}_{\mathrm{A}}$ for sample No 1 is less than 17 , indicating that there was no agglutination between anti-A and antibody in the blood sample. But the edges of $N_{B}$ and $N_{D}$ are much more than 17 i.e. agglutination occurred with anti-B and $\mathrm{D}$ respectively. So, this data indicates the $\mathrm{B}$ positive $(\mathrm{B}+)$ type blood. For the remaining data, a similar type determination is obtained. Then, for each hue, we calculated the Euclidean distance between the blood cluster and the Hemoglobin scale cluster, assuming that the closest cluster would be closest to the actual Hemoglobin value of the blood. If the Euclidean distance of two HCS colors' is equal, another situation could occur, i.e. if the RGB of a blood sample falls between two Hemoglobin values like 10 and 12, or 8 and 10 , then the average value will have considered i.e. 11 or 9 respectively.

Table 3 demonstrates anemic condition for corresponding. Hemoglobin values of HCS. This technique helps initially to indicate that a patient is anemic or not. In this second part of this document, we digitize the HCS of WHO and took a digital photograph of the blood sample after that compared them using image processing techniques. Each hue of HCS is different and their clusters for each Hemoglobin value are distinct. 
Table 2: Result analysis based on edges.

\begin{tabular}{|c|c|c|c|c|}
\hline No. of ample & $\mathbf{N}_{\mathbf{A}}$ & $\mathbf{N}_{\mathbf{B}}$ & $\mathbf{N}_{\mathbf{D}}$ & Result \\
\hline 1 & 13 & 236 & 277 & $\mathrm{~B}+$ \\
\hline 2 & 5 & 6 & 169 & $\mathrm{O}+$ \\
\hline 3 & 165 & 268 & 252 & $\mathrm{AB}+$ \\
\hline 4 & 198 & 11 & 133 & $\mathrm{~A}+$ \\
\hline 5 & 9 & 157 & 163 & $\mathrm{~B}+$ \\
\hline
\end{tabular}

Table 3: Anemic condition defined in HCS.

\begin{tabular}{|c|c|}
\hline Hemoglobin level(g/dL) & Condition \\
\hline 14 & Healthy \\
\hline 12 & Not anemic \\
\hline $8-11$ & Mild to moderate anemia \\
\hline $6-7$ & Marked anemia \\
\hline $4-5$ & Severe anemia \\
\hline Below 4 & Critical \\
\hline
\end{tabular}

Table 4: Table for measuring Euclidian distance between RGB of each Hue and RGB of Blood sample.

\begin{tabular}{|c|c|c|c|c|}
\hline \multirow{2}{*}{ Hemoglobin(g/dL) } & \multirow{2}{*}{ RGB of Scale Hue } & \multicolumn{3}{|c|}{ Euclidean Distance } \\
\cline { 3 - 5 } & & Sample 1 (143,24,16) & Sample 2 (229,31,33) & Sample 3 (162,11,6) \\
\hline 14 & $148,8,12$ & $\mathbf{1 7 . 2 3}$ & 86.78 & $\mathbf{1 5 . 5 2}$ \\
\hline 12 & $182,15,14$ & 40.07 & 53.16 & 21.91 \\
\hline 10 & $205,15,17$ & 62.66 & 32.98 & 64.56 \\
\hline 8 & $225,19,18$ & 82.18 & 19.62 & 85.63 \\
\hline 6 & $237,39,35$ & 97.07 & $\mathbf{1 1 . 4 9}$ & 98.92 \\
\hline 4 & $245,47,46$ & 108.78 & 26.10 & $\mathbf{1 4}$ \\
\hline
\end{tabular}

Table 5: Comparison of proposed method with typical Sahli's method.

\begin{tabular}{|c|c|c|c|c|}
\hline \multirow{2}{*}{ No of Sample } & \multicolumn{3}{|c|}{ Hemoglobin Detection Method } & \multirow{2}{*}{ Error } \\
\cline { 2 - 4 } & Sahli's $(\mathbf{g} / \mathbf{d L})$ & HCS $(\mathbf{g} / \mathbf{d L})$ & Proposed $(\mathbf{g} / \mathbf{d L})$ & $1.4 \%$ \\
\hline 1 & 13.8 & 14 & 14 & $6.25 \%$ \\
\hline 2 & 6.4 & 6 & 6 & $3.7 \%$ \\
\hline 3 & 13.5 & 14 & 14 & $5.66 \%$ \\
\hline 4 & 10.6 & 10 & 10 & $5.26 \%$ \\
\hline
\end{tabular}

From the RGB color space analysis of each hue, the Red intensity gives a better distinguishable value than Green intensity for calculating higher Hemoglobin levels like $12 \mathrm{gm} / \mathrm{dL}, 14 \mathrm{gm} / \mathrm{dL}$, etc, as well as for the lower Hemoglobin levels green intensities perform better. The distinct clusters of each Hemoglobin level are arranged such that the monotonicity is maintained i.e clusters of 6 Hemoglobin level comes after the next Hemoglobin level 8, 8 comes after 10, 10 comes after 12, and so on. In Table 4, the "RGB Scale Hue" column represents the RGB value of the corresponding hue. Blood samples are taken with their RGB. Hence, Euclidean distance is derived from the RGB of each hue and RGB of the blood sample. The "Euclidean Distance" column is showing the distance value of Sample 1, 2, and 3 . The minimum distance value refers to the correspondding Hemoglobin level. The minimum Euclidean distance value for sample 1 is 17.23 , so the Hemoglobin value for sample 1 is $14 \mathrm{~g} / \mathrm{dL}$. Similarly, the Hemoglobin values of samples 2 and 3 are 6 and $14 \mathrm{~g} / \mathrm{dL}$ UniversePG I www.universepg.com respectively. A comparison between our proposed model and popular standard Sahli's Hemoglobin detection method (Kharate et al., 2020; Patil et al., 2013) is representted in Table 5. Our proposed method provides the same result as the HCS scale since it is inspired by the HCS method and provides a more accurate value compared to Sahli's method. Hemoglobin values for five samples are measured from Sahli's, WHO's HCS, and the proposed method is shown in Table 5.

\section{CONCLUSION:}

In this research work, the proposed techniques are utilized for the image sets that are collected from blood samples and then processed by efficient image processing methods and algorithms. We have collected data by counting the edges for each captured image and carefully examining the data we can compute the blood group. In addition, we have computed the $\mathrm{Hb}$ level from the captured image of the blood sample by comparing it with the standard WHO's HCS scale. The experimental findings obtained from the traditional technique and 
those obtained from our proposed method are nearly identical, indicating that our proposed method provides optimal precision. Consequently, the proposed method of digital identification of blood group and $\mathrm{Hb}$ levels replaces the traditional manual lab testing process. Also, it can detect blood group and $\mathrm{Hb}$ levels quickly, easily, and with high reliability. As an android mobile phone is used here for image capturing and processing purposes, no one needs to buy an additional device for testing without three antigens (A, B, and D) and filter paper. Thereby, a cost-saving detection strategy has been possible. Thus, we can conclude that the approaches we've described are capable of detecting blood groups and Hemoglobin levels through an Android smart phone.

\section{ACKNOWLEGDEMENT:}

The authors thank the authority of the Department of Information and Communication Engineering, Pabna University of Science and Technology (PUST), for their necessary support to conduct the study.

\section{CONFLICTS OF INTEREST:}

The writers of this article stated that, there are no conflicts of the interest to publish the research article under the current issue.

\section{REFERENCES:}

1) Ahsan et al. (2017). A novel real-time non-invasive hemoglobin level detection using video images from smart phone camera. In 2017 IEEE $41^{\text {st }}$ Annual Computer Software and Applications Conference (COMPSAC). IEEE, 1, pp. 967-972. https://doi.org/10.1109/COMPSAC.2017.29

2) An et al. (2019). Integrated anemia detection and hemoglobin variant identification using point-ofcare microchip electrophoresis. Blood, 134, p. 378. https://doi.org/10.1182/blood-2019-126096

3) Azam MS, Rahman A, Iqbal SMHS, and Ahmed MT. (2020). Prediction of liver diseases by using few machine learning based approaches, Aust. $J$. Eng. Innov. Technol., 2(5), 85-90. https://doi.org/10.34104/ajeit.020.085090

4) Dhande et al. (2018). Identifying the blood group using Image Processing. Int. Res. J. Eng. Technol, 5, pp.2639-2642.

https://www.ijser.org/researchpaper/Determinationof-Blood-group-using-Image.pdf
5) Dong et al. (2017). ABO blood group detection based on image processing technology. In 2017 2nd International Conference on Image, Vision and Computing (ICIVC). IEEE, pp. 655-659. https://doi.org/10.1109/ICIVC.2017.7984637

6) Hasan et al. (2021). Geographical variation and temporal trend in anemia among children 6-59 months of age in low-and middle-income countries during 2000-2018: forecasting the 2030 SDG target. Public Health Nutrition, pp.1-20. https://doi.org/10.1017/S1368980021002482

7) Jayakumar et al. (2020). Identification and Analysis of Blood Group with Digital Microscope Using Image Processing. In IOP Conference Series: Materials Science and Engineering. IOP Publishing, 923(1), p. 012013. https://doi.org/10.1088/1757-899X/923/1/012013

8) K, M. A. and C, K. Y., (2018). Determination and Classification of Blood Groups Using Image Processing Technique. International Research J. of Engineering and Technology (IRJET), 5(5). https://www.irjet.net/archives/V5/i5/IRJET-V5I510 2.pdf

9) Kharate, B. R., et al. (2020). Estimation of hemoglobin by Sahli's and Drabkin's method. IOSR J. of Dental and Medical Sciences (IOSR-JDMS). 19(10), pp. 20-22. https://doi.org/10.9790/0853-1910122022

10) Kharkar, V.P. and Ratnaparkhe, V.R., (2013). Hemoglobin estimation methods: a review of clinical, sensor and image processing methods. Int $J$ Eng Res Technol, 2(1), pp.1-7.

11) Khatun et al. (2013). Anemia among garment factory workers in Bangladesh. Middle-East $J$. of Scientific Research, 16(4), pp.502-7. https://doi.org/10.5829/idosi.mejsr.2013.16.04.7527

12) Kumar et al. (2014). Digital WHO hemoglobin color scale: Analysis and performance. In The Sixth International Conference on EHealth, Telemedicine, and Social Medicine (ETELEMED 2014). IARIA, pp. 53-58.

13) Lewis et al. (1998). An inexpensive and reliable new hemoglobin color scale for assessing anaemia. J. of clinical pathology, 51(1), pp.21-24. http://dx.doi.org/10.1136/jcp.51.1.21

14) Marn, H. and Critchley, J.A., (2016). Accuracy of the WHO Hemoglobin Color Scale for the dia- 
gnosis of anaemia in primary health care settings in low-income countries: a systematic review and meta-analysis. The Lancet Global Health, 4(4), pp.251-265.

https://doi.org/10.1016/S2214-109X(16)00005-X

15) NHS, (2020). NSH: Health A to Z, Blood Groups. Viewed 02 August, 2021. https://www.nhs.uk/conditions/blood-groups/

16) Patil et al. (2013). Variability and accuracy of Sahli's method in estimation of hemoglobin concentration. Natl J Integr Res Med, 4(1), pp.38-44. https://www.researchgate.net/publication/26674197 $\underline{6}$

17) Ravindran et al. (2017). Determination and classification of blood types using image processing techniques. International J. of Computer
Applications, 157(1), pp.12-16.

18) Stott, G.J. and Lewis, S.M. (1995). A simple and reliable method for estimating hemoglobin. Bulletin of the World Health Organization (WHO), 73(3), p.369.

https://www.ncbi.nlm.nih.gov/pmc/articles/PMC248 6662/

19) Vala, H.J. and Baxi, A., (2013). A review on Otsu image segmentation algorithm. International J. of Advanced Research in Computer Engineering \& Technology (IJARCET), 2(2), pp.387-389.

20) Zafar et al. (2018). Plasmonics-based refractive index sensor for detection of hemoglobin concentration. IEEE Sensors Journal, 18(11), pp. 1-1. https://doi.org/10.1109/JSEN.2018.2826040 3(5), 73-81. https://doi.org/10.34104/ajeit.021.073081 @ @) 